\title{
Shedding Light on Endometriosis: Patient and Provider Perspectives on a Challenging Disease
}

\author{
Madalene Zale \\ Thomas Jefferson University \\ Emily Lambert \\ Thomas Jefferson University \\ Marianna LaNoue \\ Thomas Jefferson University \\ Amy E. Leader \\ Thomas Jefferson University
}

Follow this and additional works at: https://jdc.jefferson.edu/nursfp

Part of the Nursing Commons, Obstetrics and Gynecology Commons, and the Women's Health Commons

Let us know how access to this document benefits you

\section{Recommended Citation}

Zale, Madalene; Lambert, Emily; LaNoue, Marianna; and Leader, Amy E., "Shedding Light on Endometriosis: Patient and Provider Perspectives on a Challenging Disease" (2020). College of Nursing Faculty Papers \& Presentations. Paper 103.

https://jdc.jefferson.edu/nursfp/103

This Article is brought to you for free and open access by the Jefferson Digital Commons. The Jefferson Digital Commons is a service of Thomas Jefferson University's Center for Teaching and Learning (CTL). The Commons is a showcase for Jefferson books and journals, peer-reviewed scholarly publications, unique historical collections from the University archives, and teaching tools. The Jefferson Digital Commons allows researchers and interested readers anywhere in the world to learn about and keep up to date with Jefferson scholarship. This article has been accepted for inclusion in College of Nursing Faculty Papers \& Presentations by an authorized administrator of the Jefferson Digital Commons. For more information, please contact: JeffersonDigitalCommons@jefferson.edu. 


\section{INTRODUCTION}

Endometriosis is a disease defined as the presence of endometrial-like tissue found outside of the uterus (1). It affects an estimated one in 10, and approximately 176 million, women worldwide, making it one of the most prevalent diseases in gynecologic practice $(2,3,4)$. It is most frequently associated with chronic pelvic pain, dysmenorrhea, menorrhagia, and dyspareunia, and is a leading cause of infertility $(5,6)$. Current guidelines for treatment and management of the disease include oral contraceptives and hormone replacement therapy as options for pain relief, as well as surgical management when medical management has otherwise failed (7). Presently, there is no known cure for endometriosis, and while it's likely that there is a genetic component, there is no known cause (3).

Individuals with endometriosis are often misdiagnosed or encounter a delayed diagnosis, which leads to unnecessary and inappropriate treatment measures as well as additional costs to both patients and health systems $(8,9)$. Research shows that individuals with endometriosis are three and a half times more likely to receive a diagnosis of Irritable Bowel Syndrome than those without an endometriosis diagnosis (8). Furthermore, a study evaluating active-duty women found that lost time due to endometriosis-related disabilities over six years equated to 21,746 days, at a cost of $\$ 2.6$ million (9). In terms of quality of life, studies have found widespread detrimental suffering on physical, emotional, and social levels (10). Earlier detection and diagnosis would allow for more appropriate treatment options, minimize costs, and reduce years of under-treated, painful symptoms.

We conducted a mixed-methods study with two primary aims: (1) to assess perceptions and awareness of endometriosis among providers who diagnose and treat endometriosis (2) to assess the endometriosis patient experience in the United States. We sought to identify relevant 
themes that contributed to understanding the experiences of those who either have endometriosis or are involved in its treatment.

\section{METHODS}

\section{Study Design}

Providers completed a brief survey related to their understanding of the prevalence of endometriosis; a sample of these providers were interviewed about their experiences in diagnosing and treating it. Individuals with endometriosis were invited to participate in an interview in order to understand their experiences of living with the disease. The home institution's Institutional Review Board approved the study. Providers and patients provided verbal consent prior to data collection. All interviews were conducted via telephone, audiorecorded, and transcribed verbatim.

\section{Recruitment of Participants}

We recruited providers by introducing the study at the home institution's conferences and seminars in gastroenterology, obstetrics and gynecology, internal medicine and primary care. We recruited patients for interviews by collaborating with a national, non-profit endometriosis organization that posted a recruitment flyer on their social media pages.

\section{Data Analysis}

The proportion of providers responding correctly to the endometriosis prevalence question was calculated and compared across provider specialties, number of years in practice and gender. Demographic characteristics of the providers were analyzed by calculating means and frequencies. All quantitative analyses were conducted using SPSS. For provider and patient interviews, codebooks were created and used to analyze responses from interviews to identify recurring themes. Two coders independently coded each transcript and discrepancies were resolved through consensus and with a third member of the research team. We used a thematic 
analysis approach to code the interviews; all major themes and sub-themes were derived from the data and none were identified in advance (11).

\section{RESULTS}

Participants

Fifty-three providers completed the survey. The response rate was $27 \%$; all surveys had complete data. Descriptive characteristics of providers are displayed in Table 1.

Quantitative Results: Provider Understanding of the Prevalence of Endometriosis

Of the 53 providers who completed the survey, only six (11\%) identified the correct prevalence of endometriosis ( 1 in 10). Of those six providers, five were OB/GYN's and one was a gastroenterologist; five were female; five were physicians and one was a nurse practitioner. Additionally, four spent $76-100 \%$ of their time in direct patient care; one spent $51-75 \%$; and one spent $<50 \%$. Unequal distribution of cells and a small sample size precluded any further bivariate analyses.

Qualitative Results: Provider Interviews

Four of the 53 providers participated in an interview (average interview time $=17$

minutes). Interviews uncovered several key themes including limited training and the difficulty of making a diagnosis, patient-provider dynamic and the referral process, and the importance of multidisciplinary collaboration (Tables 2 and 3).

\section{Limited Training and Difficult Diagnosis}

Throughout the interviews, it became evident that the etiology, or diagnosis of endometriosis, was not covered comprehensively in any of the participant's respective clinical training. Endometriosis is often considered a "benign" female disease because it is noncancerous and presents with diffuse symptoms in various parts of the body without a definitive cause. 
Therefore, it is typically not emphasized nor given adequate coverage during training. Both of the aforementioned items tied into an overarching explanation for the overall low estimated prevalence by the four providers interviewed and their colleagues who were queried in the survey. There was unanimity among providers that establishing an endometriosis diagnosis is difficult on account of a myriad of factors, including the fact that surgery is required, imaging is necessary, and pelvic pain is unspecified.

\section{Provider-Patient Dynamic and the Referral Process}

Each of the providers acknowledged the importance of establishing a relationship with their patients. They explained different aspects, including open and honest communication, documenting an accurate patient history, and working towards empowering the patient. Similarly, all of the participants noted that the referral process plays an integral role in their overall treatment plan, while mentioning that not referring to a specialist to effectively treat the disease can ultimately hinder patient outcomes.

\section{Importance of Multidisciplinary Collaboration}

The providers ultimately agreed that because the disease presents in a variety of ways, it is critical that the entire care team is in agreement of the treatment plan. They articulated that having clear pathways for interdisciplinary collaboration makes for more timely and accurate diagnoses. Providers suggested ways to foster and bolster collaboration and close gaps in care, including having multidisciplinary care teams and endometriosis diagnosis and treatment guidelines, inclusive of numerous specialties.

Qualitative Results: Patient Interviews

Twelve patients completed interviews about their experience with endometriosis (average interview time $=31$ minutes). Their characteristics are described in Table 4 . 
The patient interviews revealed several themes, including the psychological impact of endometriosis, difficulties interacting with the healthcare system, the financial impact of the disease, being averse to hormonal therapies despite limited treatment options, lack of awareness and self-advocacy, concerns about fertility, and quality of life (Tables 5 and 6).

\section{Psychological Impact}

Each participant interviewed spoke about the psychological aspect of this physical disease. Pain, misdiagnoses, and limited treatment options all had a significant impact on these individual's mental health. The stigma of having endometriosis paired with having to deal with the psychological--related symptoms stemmed from years of symptoms being dismissed and pain being delegitimized. .

\section{Difficulties Interacting with the Health Care System}

Every patient talked about struggling with providers and feeling frustrated with the healthcare system. Patients noted that they encountered providers who lacked the skills and knowledge to properly manage the disease. Similarly, patients reported feeling patronized by providers during their respective endometriosis journeys. And whether it was before they received an official diagnosis or the years after, patients felt strongly that their concerns had not been heard.

\section{The Financial Impact}

Several participants shared the financial impact of the disease. Some said that endometriosis limited the types of careers they could have, and others mentioned the difficulties of working and managing their symptoms. Additionally, participants discussed how the disease impacted employment status, as in being limited to jobs with full health benefits, or having to leave positions on account of time off restrictions. Interviewees noted financial constraints 
related to treatment options, from caps on insurance allotments for physical therapy, to high outof-pocket costs for certain prescription medications, like Lupron. And despite the fact that all of the participants were insured, the particulars of the disease's treatment options still contributed to a significant economic burden that had a negative effect on overall quality of life.

\section{Being Averse to Hormonal Therapy and Limited Treatment Options}

Another predominant theme among patients was being averse to hormonal therapies, despite being the only non-surgical option for the treatment and management of endometriosis. Whether participants opposed taking hormonal therapy on account of preference, side effects, or fertility concerns, most expressed strong dislike and frustration in knowing that it remains one of the main treatment options. Similarly, participants expressed their concerns about the overall lack of treatment options, despite the high prevalence of the disease.

\section{Lack of Awareness among Clinicians and the Need for Self-Advocacy}

Many patients felt that clinicians lacked a general awareness of the disease and expressed frustration because of the expectation that clinicians have expertise in the diseases that they treat. As a result of providers' lack of training in endometriosis, many patients ultimately became their own advocate after years of being dismissed, ignored, and misdiagnosed. Once patients had conducted their own extensive research, in addition to seeking additional medical opinions, only then were they able to successfully move forward in their proper disease management.

\section{Fertility Concerns}

The impact that endometriosis has on fertility specifically is multi-faceted. For many patients, preserving one's fertility was critical and they expressed the various challenges related to having children in conjunction with their endometriosis. For some of, their endometriosis was discovered as a result of the inability to conceive, for others, diagnosis with the disease pre- 
empted the conversation about fertility. The patients had conversations about fertility with their healthcare providers and partners, because it not only impacted their own health and well-being but also personal relationships. Participants spoke about having to take time consuming, expensive steps (which are not covered by insurance), to preserve fertility. Moreover, interviewees explained how they actually ended up becoming sicker after treatments to facilitate the conceiving process, (i.e., from in vitro fertilization).

\section{Quality of Life}

Throughout the course of the interviews, it became apparent that endometriosis has a significant impact on quality of life. Many patients mentioned its limiting and detrimental effects, including day-to-day activities, work, school, relationships, and social life. Additionally, it should be noted that the psychological and financial aspects of the disease had the most profound impact on overall quality of life. Most often patients' quality of life is dictated by their financial resources, which in turn can lead patients to despair, having a negative psychological impact. This ends up becoming an extremely difficult to manage continuous cycle.

\section{DISCUSSION}

The purpose of this study was to contribute to the understanding of the experience of individuals who either have endometriosis or are involved in treating it. Our research revealed a lack of awareness of the disease among providers, which can be attributed to a general lack of training in both school and practice, and subsequently to a wide misperception of the actual prevalence of the disease. Additionally, we found that endometriosis can be particularly difficult to diagnose, which while consistent with the literature, is worth reiterating that it does in fact complicate the pathway to a timely and proper diagnosis. 
In our study, only $11 \%$ of providers correctly identified the prevalence of endometriosis. This gap in knowledge has implications for patients, who noted the lack of understanding among clinicians tasked with treating endometriosis. Frequently, patients felt that their diagnosis may have been delayed due to unnecessary tests and referrals, often because providers were unsure about how to diagnose or treat the disease. And while the number of providers interviewed is relatively small $(n=4)$, it's fair to say that these themes are common in the literature, which can afford further reliability to the small sample findings. In particular, our findings are similar to a study that examined provider knowledge and treatment strategies for endometriosis among a group of Dutch general practitioners (GPs) (12). The results of the study found that GPs did not immediately recognize the symptoms that were caused by endometriosis, which ultimately contributed to diagnostic delay. Moreover, 77\% of GPs surveyed indicated that they needed more education on endometriosis (12). Creating ongoing educational opportunities for medical professionals would not only increase awareness but also enhance timely diagnosis and treatment.

Findings from our study also highlighted the difficulty of diagnosing and treating endometriosis. For a disease that affects hundreds of millions of individuals, and presents across multiple clinical specialties, it's of interest and concern that the disease itself, its symptoms, and the management of, is not covered in more detail in academic training in any capacity. Furthermore, the research that does exist is not only limited, but makes it very clear that better diagnostic measures are needed (13). Available treatment and management tools for endometriosis are insufficient. As a result, individuals with endometriosis must deal with a system that is often ill-equipped to correctly diagnose and treat the condition, which only further 
contributes to the negative social and psychological impact of endometriosis on patient's overall quality of life (14).

Our approach to understanding endometriosis was particularly meaningful because of its study design. Our dual evaluation method allowed for a direct comparison of any pertinent similarities or differences that existed between the two participant groups. Our analysis of the provider experience juxtaposed with the patient experience provided a unique perspective to closely dissect not only providers' responses to the survey questions but also how the disease is treated from those directly involved in the process. At the same time, we were able to evaluate the patient experience and those factors that contribute to their respective experiences with the disease including, symptom, diagnosis, management trajectory, and self-advocacy and management measures. This last theme is a common finding, particularly as it relates to the notion of empowerment; moreover it leads to the question as to if the medical community's failure to diagnose and/or effectively treat the illness should serve as a catalyst for empowering patients and the potential downside associated with 'self-management' (15).

Despite the continued difficulties in diagnosing and treating endometriosis, progress is being made in efforts to increase awareness and better understand the disease. While our study is based solely on the US experience, international efforts have been successful in addressing many of issues identified herein. For example, in the UK there are now a number of British Society for Gynaecological Endoscopy centres of excellence in endometriosis care, and clinical guidelines from the National Institute for Health Care and Excellence in the UK were created and published in $2017(16,17)$. Similarly, in more recent years in the United States, community action has also invoked system-level changes. Presently there is a proposed bill for legislation (S. 8543/A. 10763) that would make endometriosis and menstrual health a mandatory part of New York State 
middle and high school curriculum (18). The bill is the result of the efforts of the Endometriosis Foundation of America, medical and public health professionals, and bi-partisan elected representatives (19).

Another key finding from our research was the financial burden of the disease. While "financial toxicity" is most often associated with the extreme costs of being treated for cancer, it also can be extended to those patients with endometriosis (20). Our study found that having endometriosis could restrict an individual's career path, in terms of the types of jobs and positions that may be feasible. The disease also impacted insurance coverage, such that treatment options were often capped to a certain number and/or didn't include certain treatments. A similar study found that patients commonly reported restrictions on coverage, including limitations to a certain number of procedures, as well as pre-set limits related to endometriosis in overall healthcare costs. Participants also reported being denied full coverage for endometriosis-related treatments and procedures since they were often erroneously considered infertility treatments (21). While these specific restrictions are unique to the U.S., findings of financial toxicity in the realm of endometriosis in this study are important because international studies have directly linked this same concept to an individual's overall health-related quality of life as well (22).

Lastly, our research highlighted the fact that treatment options for endometriosis are extremely limited. For a disease that affects so many, it is unfortunate that the only non-surgical option for treatment (i.e., hormonal therapy) is one that is so unfavorable among patients. Hormonal therapy is consistently offered as a first-line treatment despite the fact that endometriosis can present with myriad symptoms and widespread effects. Our findings, paired with clinical evidence that birth control may be insufficient to improve endometriosis symptoms, 
further reiterate just how critical it is that new treatment options be researched, considered, and made available (23).

The results of our study have far-reaching implications for clinicians and researchers who are tasked with treating this complicated and multimodal disease. Revising the current guidelines related to treating endometriosis, i.e., creating coding for endometriosis excision techniques; having a multi-specialty group of guidelines for addressing pelvic pain; developments in research; securing funding; and advocating for policy shift, among others, should be considered when looking to next steps for advancing the overall standards of care related to endometriosis. On a more granular level, this means holding discussions with the endometriosis community to incorporate patients, advocates, nonprofit leaders, and medical experts to best meet the needs of the patient population.

\section{LIMITATIONS}

This exploratory study consisted of a small patient sample that, while geographically diverse, was somewhat homogenous racially and ethnically. Our findings are unlikely to represent all of the perspectives of patients with endometriosis or the providers who treat them. We only included patients who had a surgical confirmation of endometriosis, and therefore our study did not include those without a definitive surgical diagnosis or asymptomatic individuals. Additionally, the study consisted of a small sample of providers as well. For both samples, our data is dependent on participants' recall bias and on selection bias, since only the responses of those who volunteered to participate are represented here. 